\title{
INFLUÊNCIA DOS PARÂMETROS DE PROCESSO NA OCORRÊNCIA DE ROMBOIDADE EM TARUGOS DE AÇO DE MÉDIO TEOR DE CARBONO PRODUZIDOS NA ARCELORMITTAL CARIACICA*
}

\begin{abstract}
Hiury Voltz ${ }^{1}$ Erasmo Schultz ${ }^{2}$ Miguel Arcanjo Freitas Almeida ${ }^{3}$ Estéfano Aparecido Vieira ${ }^{4}$

Resumo

Diversos são os defeitos que ocorrem durante o lingotamento contínuo dos aços. Um tipo de defeito de forma recorrente encontrado no lingotamento contínuo de jato aberto da ArcelorMittal Cariacica é a romboidade. O foco deste trabalho foi avaliar a influência da velocidade da água de refrigeração do molde, da velocidade de lingotamento, da lubrificação, do superheat e da conicidade na ocorrência de romboidade em aços de médio teor de carbono. Por meio da técnica de planejamento de experimentos, testes foram realizados para melhor compreender os efeitos de cada um dos fatores listados. Após análise estatística feita no MINITAB ${ }^{\circledR}$ versão 16.0, os resultados mostraram que a melhor condição, ou seja, as menores romboidades, foram encontradas reduzindo-se o superheat e aumentando-se a velocidade da água de refrigeração do molde, a velocidade de lingotamento e a taxa de lubrificação. O uso de moldes com menor número de corridas foi também outro fator que favoreceu a redução deste tipo de defeito.
\end{abstract}

Palavras-chave: Romboidade; Lingotamento contínuo; Tarugos; Planejamento de experimento.

\section{INFLUENCE OF PROCESS PARAMETERS IN THE OCCURRENCE OF RHOMBOIDITY IN STEEL BILLETS OF MEDIUM CARBON PRODUCED AT ARCELORMITTAL CARIACICA}

\begin{abstract}
There are several defects which occur during the continuous casting of steels. One type of defect recurrently found in the open-stream continuous casting of ArcelorMittal Cariacica is the rhomboidity. The focus of the present investigation was to evaluate the influence of the velocity of mold cooling water, casting speed, lubrication, superheat and mould taper on the event of rhomboidity in medium-carbon steel. Through Design of Experiments, tests were conducted to better understand the effects of each of the factors listed. After statistical analysis in MINITAB® 16.0, the results showed that the best condition, that is, lower rhomboiditys were found reducing the superheat and increasing the speed of mold cooling water, casting speed, and lubrication rate. The use of moulds with reduced number of tap was a favorable factor to reduce this type of flaw.
\end{abstract}

Keywords: Rhomboidity; Continuous casting; Billets; Design of experiments.

1 Engenheiro Metalurgista e Mestrando em Engenharia Metalúrgica e de Materiais do Programa de Pós-Graduação em Engenharia Metalúrgica e de Materiais, PROPEMM, Vitória, ES, Brasil.

2 Tecnólogo em Metalurgia e Materiais, Supervisor de Produção do Lingotamento Contínuo da ArcelorMittal Cariacica, Cariacica, ES, Brasil.

3 Engenheiro Metalurgista, Engenheiro de Processos da Aciaria da ArcelorMittal Cariacica, Cariacica, ES, Brasil.

4 Prof. Dr. do Instituto Federal do Espírito Santo, IFES, dos cursos de graduação e pós-Graduação em Engenharia Metalúrgica e de Materiais, Vitória, ES, Brasil. 


\section{INTRODUÇÃO}

Um tipo de defeito recorrente encontrado em lingotamentos contínuos de jato aberto é a romboidade que é detectada através de inspeção visual ou com o emprego de instrumentos de medidas [1]. A romboidade é afetada por diversos fatores e pode ser definida como um defeito de forma do tarugo produzido via lingotamento contínuo no qual sua seção quadrada sofre uma distorção apresentando uma diagonal maior que a outra (Figura 1).

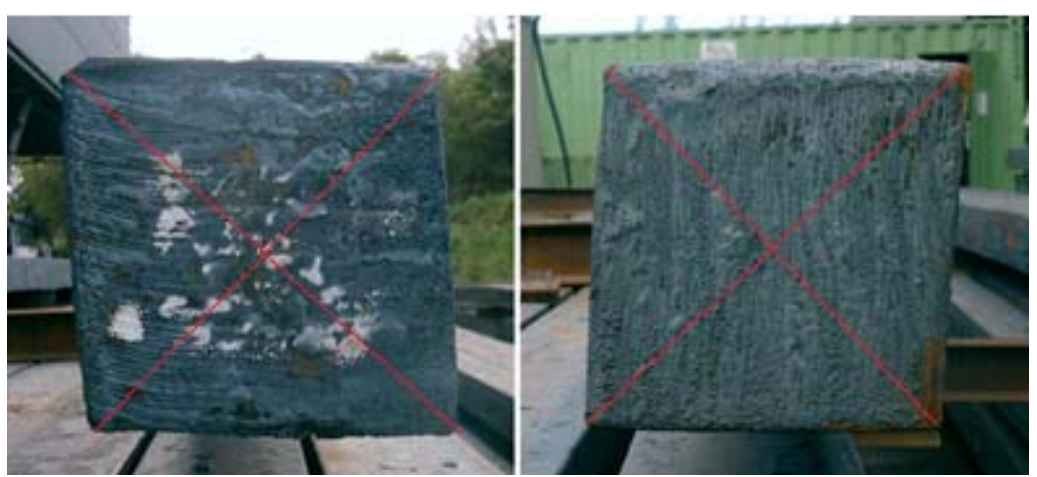

Figura 1 - Tarugos com incidência de romboidade produzidos na ArcelorMittal Cariacica.

A romboidade severa causa dificuldades na movimentação dos tarugos no reaquecimento, particularmente nos fornos empurradores. Na laminação, dobras nos cantos podem ocorrer gerando costuras no produto final. Além disso, outros defeitos, como, por exemplo, as trincas diagonais, ocorrem associados à romboidade [2].

A romboidade é considerada severa se a diferença entre as diagonais ultrapassar $6 \mathrm{~mm}$ [2]. Na ArcelorMittal Cariacica, tarugos com até $9 \mathrm{~mm}$ de romboidade são liberados, entre 10 e $13 \mathrm{~mm}$ o aproveitamento é avaliado pela engenharia de processo e acima de $13 \mathrm{~mm}$ são sucateados diretamente.

Tendo em vista as circunstâncias acima citadas, neste trabalho, fez-se um planejamento de experimentos visando avaliar a influência de alguns fatores e de suas interações na ocorrência de romboidade em aços de médio teor carbono produzidos via lingotamento contínuo na ArcelorMittal Cariacica.

\subsection{Fatores que Afetam a Formação da Romboidade}

O teor de carbono presente nos aços pode favorecer o surgimento da romboidade, sendo que os aços de médio teor de carbono parecem ser mais sensíveis do que os aços de baixo ou alto carbono. Diferentemente dos aços de baixo teor de carbono que apresentam baixa extração de calor no molde, os aços de médio teor de carbono exprimem elevada taxa de transferência de calor no menisco por não apresentarem uma pele rugosa originária da reação peritética $(\% C=0,09$ a 0,17$)$. Por outro lado, também possuem uma pequena faixa de solidificação, ou seja, a distância entre $\mathrm{T}_{\text {liquidus }}-\mathrm{T}_{\text {solidus }}$ é de 40 a $50^{\circ} \mathrm{C}$, ao passo que nos aços de alto teor de carbono a faixa de solidificação é bem maior (por exemplo, cerca de $100^{\circ} \mathrm{C}$ ). Assim, o impacto do resfriamento não uniforme ao gerar uma casca solidificada não uniforme é maior para os aços médio teor de carbono [1]. 
Além do teor de carbono, todos os fatores que contribuem para a distorção do molde e o aumento do gap de ar, que é o meio de maior resistência à transferência de calor entre a pele solidificada e a parede do molde, são classificados como de extrema importância para minimizar problemas de romboidade, pois promovem uma extração de calor não uniforme e a formação de uma casca solidificada irregular e propensa à romboidade. Dessa forma, fatores como velocidade, pressão e qualidade da água de refrigeração do molde, oscilação do molde, lubrificação, variação de nível de metal no molde, velocidade de lingotamento, superheat, composição e espessura da parede do molde, tipo de suporte do molde, conicidade, resfriamento secundário afetam diretamente a formação da romboidade.

Neste sentido, recomenda-se a operação com "moldes frios", que é obtida com altas velocidades de passagem de água no canal de refrigeração (14 a $15 \mathrm{~m} / \mathrm{s}$ ) e temperaturas de entrada da água no molde em torno de $30^{\circ} \mathrm{C}$. Os moldes, quando resfriados adequadamente, não apresentam fervura intermitente de água no canal de refrigeração e, consequentemente, apresentam temperatura da face quente (interna) abaixo da temperatura de recristalização do cobre (temperatura de amolecimento do cobre), garantindo menor deformação na região do menisco [1].

Taxas de fluxo de óleo de 20 a $30 \mathrm{ml} / \mathrm{min}$ são necessárias para a adequada, mas não excessiva, lubrificação do molde [3]. A interrupção do fluxo de óleo na região do menisco pode ser provocada pela distribuição não uniforme de óleo lubrificante nas diferentes faces do molde.

As variações de nível de aço no molde são aleatórias, podendo diferir (subida e descida do nível) não só nas quatro faces, mas também através de uma dada face. Em operações de "molde quente", no qual a temperatura máxima do molde situa-se próxima ao intervalo de ebulição do óleo, a região abaixo do menisco estaria relativamente "seca" (com pouco óleo líquido). Assim, devido a essa aleatoriedade, se o menisco sobe, a temperatura máxima do molde o acompanha da região com pouco óleo para outra com óleo líquido. Quando isso acontece, o óleo vaporiza e a transferência de calor cresce $20 \%$. Por outro lado, quando o nível de metal desce, a temperatura máxima do molde desloca-se para uma região com pouco óleo. Neste caso, quase não haverá aumento da transferência de calor. Portanto, quando o nível de metal sobe em uma parte e desce em outra, variações significativas na transferência de calor ocorrem, proporcionando diferenças na espessura da casca solidificada e contribuindo para a incidência de romboidade [1].

Maiores velocidades de lingotamento proporcionam um menor tempo de residência do aço no molde, o que resulta em menores espessuras da casca solidificada, a qual oferecerá menor resistência à pressão ferrostática, deformando-se e reduzindo o gap de ar. Além disso, em temperaturas mais elevadas, há uma menor contração térmica da casca solidificada, o que melhora o contato molde/tarugo e não contribui para o tamanho do gap [4].

Um superheat maior afetará a espessura dos cantos da casca solodificada, tornando o lingote mais susceptível a defeitos como romboidade [4].

Conicidades mais acentuadas (superiores a $2 \% / \mathrm{m}$ ) são recomendadas no topo do molde de tal forma a reduzir a conicidade negativa provocada pela distorção térmica das paredes do molde [6]; por outro lado, abaixo da região do menisco até próximo à saída, a conicidade deve ser cada vez menos pronunciada, - entre 0,6 e 2,0\%/m pois apenas a contração devido à solidificação deve ser levada em consideração [5]. 


\section{MATERIAIS E MÉTODOS}

No presente estudo, optou-se por planejamentos de experimentos do tipo fatorial, em que foram estabelecidos dois níveis para cada fator. Desta forma, trabalhou-se com um planejamento fatorial $2^{\mathrm{k}}$, onde $\mathrm{K}$ representa o número de fatores a serem testados em cada experimento. Entre todos os fatores, os selecionados para a realização de experimentos foram velocidade da água de refrigeração do molde, velocidade de lingotamento, lubrificação, superheat e conicidade. A romboidade foi determinada através da diferença das diagonais medidas com o auxílio de um paquímetro devidamente calibrado. As medições foram feitas em quatro regiões do tarugo - duas na região central e uma em cada extremidade conforme mostra a figura 2 esquematicamente

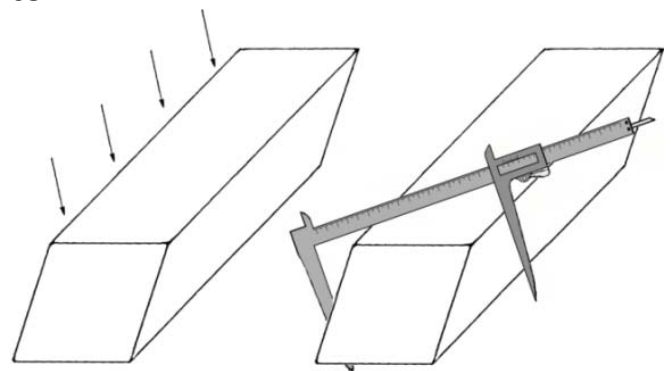

Figura 2 - Representação esquemática do processo de medição das diagonais dos tarugos.

Em virtude de limitações impostas pelo processo produtivo, os experimentos foram realizados testando-se dois ou três fatores por vez, sendo criadas, portanto, quatro ou oito combinações respectivamente $\left(2^{k}, k=2\right.$ ou $\left.k=3\right)$ dentro de uma mesma corrida. Neste caso, os fatores escolhidos foram velocidade da água de refrigeração do molde (utilização de vazões de água de cerca de 1750 e 1500 L/min que proporcionam velocidades de cerca de 13 e $11 \mathrm{~m} / \mathrm{s}$ respectivamente), lubrificação (utilização de fluxos de lubrificação de 140 e $80 \mathrm{~mL} / \mathrm{t}$ de aço que fornecem taxas de lubrificação nos intervalos de 42 - 58 e 25 - $33 \mathrm{~mL} / \mathrm{min}$ ) e velocidade de lingotamento (utilização de válvulas calibradas de troca rápida de $\varnothing 18,0$ e $\varnothing 15,5$ $\mathrm{mm}$ que fornecem velocidades aproximadas de 3,2 e $2,4 \mathrm{~m} / \mathrm{min}$ respectivamente), pois são os parâmetros que mais rapidamente respondem às mudanças feitas durante o processo.

Para verificar a influência da conicidade e do superheat foi necessária a realização de planejamento fatorial $2^{k}$ em blocos. Para a conicidade, foram feitos experimentos em dois veios; em um dos veios foi utilizado um molde com menos de 100 corridas (molde com conicidade nominal) e em outro veio, um molde com mais de 300 corridas (molde com conicidade adequada, porém com certo desgaste e apresentando distorção). Para o superheat, os testes foram feitos em diferentes corridas, uma apresentando um alto superheat $\left(55-75^{\circ} \mathrm{C}\right)$ e outra com um baixo superheat $\left(25-45{ }^{\circ} \mathrm{C}\right)$. Nas tabelas 1,2 e 3 são apresentadas as matrizes experimentais dos experimentos 1, 2 e 3, respectivamente.

Para a realização da análise estatística dos dados foi utilizado o software MINITAB ${ }^{\circledR}$ versão 16.0, bastante difundido no meio acadêmico e utilizado na ArcelorMittal Cariacica. O nível de significância utilizado foi de 15\%. A opção por este valor devese ao dinamismo dos experimentos industriais, consequentemente, à dificuldade de se manter as mesmas condições de processo entre experimentos. A análise dos experimentos foi feita avaliando-se o efeito das interações entre os fatores por meio do gráfico de Pareto para os efeitos e do gráfico multi-vari. 
Tabela 1. Matriz experimental do experimento 1 (Influência do fluxo de lubrificação).

\begin{tabular}{ccccc}
\hline Teste & $\begin{array}{c}\text { Válvula calibrada } \\
\text { de troca rápida }\end{array}$ & $\begin{array}{c}\text { Vazão da água de } \\
\text { refrigeração do molde }\end{array}$ & $\begin{array}{c}\text { Fluxo de } \\
\text { lubrificação }\end{array}$ & $\begin{array}{c}\text { Quantidade } \\
\text { de amostras }\end{array}$ \\
\hline $\mathrm{A}$ & $\varnothing 18,0 \mathrm{~mm}$ & $1750 \mathrm{~L} / \mathrm{min}$ & $140 \mathrm{~mL} / \mathrm{t}$ de aço & 4 \\
$\mathrm{~B}$ & $\varnothing 18,0 \mathrm{~mm}$ & $1750 \mathrm{~L} / \mathrm{min}$ & $80 \mathrm{~mL} / \mathrm{t}$ de aço & 6 \\
$\mathrm{C}$ & $\varnothing 18,0 \mathrm{~mm}$ & $1500 \mathrm{~L} / \mathrm{min}$ & $80 \mathrm{~mL} / \mathrm{t}$ de aço & 4 \\
$\mathrm{D}$ & $\varnothing 18,0 \mathrm{~mm}$ & $1500 \mathrm{~L} / \mathrm{min}$ & $140 \mathrm{~mL} / \mathrm{t}$ de aço & 4 \\
$\mathrm{E}$ & $\varnothing 15,5 \mathrm{~mm}$ & $1500 \mathrm{~L} / \mathrm{min}$ & $140 \mathrm{~mL} / \mathrm{t}$ de aço & 4 \\
$\mathrm{~F}$ & $\varnothing 15,5 \mathrm{~mm}$ & $1500 \mathrm{~L} / \mathrm{min}$ & $80 \mathrm{~mL} / \mathrm{t}$ de aço & 2 \\
$\mathrm{G}$ & $\varnothing 15,5 \mathrm{~mm}$ & $1750 \mathrm{~L} / \mathrm{min}$ & $80 \mathrm{~mL} / \mathrm{t}$ de aço & 6 \\
$\mathrm{H}$ & $\varnothing 15,5 \mathrm{~mm}$ & $1750 \mathrm{~L} / \mathrm{min}$ & $140 \mathrm{~mL} / \mathrm{t}$ de aço & 4 \\
\hline
\end{tabular}

Tabela 2. Matriz experimental do experimento 2 (Influência do superheat).

\begin{tabular}{ccccc}
\hline Teste & $\begin{array}{c}\text { Válvula calibrada } \\
\text { de troca rápida }\end{array}$ & $\begin{array}{c}\text { Vazão da água de } \\
\text { refrigeração do molde }\end{array}$ & Superheat & $\begin{array}{c}\text { Quantidade } \\
\text { de amostras }\end{array}$ \\
\hline $\mathrm{A}$ & $\varnothing 18,0 \mathrm{~mm}$ & $1750 \mathrm{~L} / \mathrm{min}$ & Alto & 4 \\
$\mathrm{~B}$ & $\varnothing 18,0 \mathrm{~mm}$ & $1500 \mathrm{~L} / \mathrm{min}$ & Alto & 4 \\
$\mathrm{C}$ & $\varnothing 15,5 \mathrm{~mm}$ & $1500 \mathrm{~L} / \mathrm{min}$ & Alto & 4 \\
$\mathrm{D}$ & $\varnothing 15,5 \mathrm{~mm}$ & $1750 \mathrm{~L} / \mathrm{min}$ & Alto & 5 \\
$\mathrm{E}$ & $\varnothing 15,5 \mathrm{~mm}$ & $1750 \mathrm{~L} / \mathrm{min}$ & Baixo & 4 \\
$\mathrm{~F}$ & $\varnothing 15,5 \mathrm{~mm}$ & $1500 \mathrm{~L} / \mathrm{min}$ & Baixo & 4 \\
$\mathrm{G}$ & $\varnothing 18,0 \mathrm{~mm}$ & $1500 \mathrm{~L} / \mathrm{min}$ & Baixo & 5 \\
$\mathrm{H}$ & $\varnothing 18,0 \mathrm{~mm}$ & $1750 \mathrm{~L} / \mathrm{min}$ & Baixo & 5 \\
\hline
\end{tabular}

Tabela 3. Matriz experimental do experimento 3 (Influência do número de corridas no molde).

\begin{tabular}{cccccccc}
\hline Teste & $\begin{array}{c}\text { Válvula de } \\
\text { troca } \\
\text { rápida }\end{array}$ & $\begin{array}{c}\text { Vazão da } \\
\text { água do } \\
\text { molde }\end{array}$ & $\begin{array}{c}\text { Fluxo de } \\
\text { lubrificação }\end{array}$ & $\begin{array}{c}\text { Número corridas } \\
\text { do molde } \\
\text { Veio 2 }\end{array}$ & \multicolumn{2}{c}{$\begin{array}{c}\text { Quantidade de } \\
\text { amostras 3 }\end{array}$} \\
\hline $\mathrm{A}$ & $\varnothing 18,0 \mathrm{~mm}$ & $1500 \mathrm{~L} / \mathrm{min}$ & $140 \mathrm{~mL} / \mathrm{t}$ & $<100$ & $>300$ & 3 & 3 \\
Veio 2 & Veio 3 \\
$\mathrm{B}$ & $\varnothing 18,0 \mathrm{~mm}$ & $1500 \mathrm{~L} / \mathrm{min}$ & $80 \mathrm{~mL} / \mathrm{t}$ & $<100$ & $>300$ & 4 & 4 \\
$\mathrm{C}$ & $\varnothing 15,5 \mathrm{~mm}$ & $1500 \mathrm{~L} / \mathrm{min}$ & $80 \mathrm{~mL} / \mathrm{t}$ & $<100$ & $>300$ & 3 & 4 \\
$\mathrm{D}$ & $\varnothing 15,5 \mathrm{~mm}$ & $1500 \mathrm{~L} / \mathrm{min}$ & $140 \mathrm{~mL} / \mathrm{t}$ & $<100$ & $>300$ & 4 & 4 \\
$\mathrm{E}$ & $\varnothing 15,5 \mathrm{~mm}$ & $1750 \mathrm{~L} / \mathrm{min}$ & $140 \mathrm{~mL} / \mathrm{t}$ & $<100$ & $>300$ & 2 & 2 \\
$\mathrm{~F}$ & $\varnothing 15,5 \mathrm{~mm}$ & $1750 \mathrm{~L} / \mathrm{min}$ & $80 \mathrm{~mL} / \mathrm{t}$ & $<100$ & $>300$ & 4 & 5 \\
$\mathrm{G}$ & $\varnothing 18,0 \mathrm{~mm}$ & $1750 \mathrm{~L} / \mathrm{min}$ & $80 \mathrm{~mL} / \mathrm{t}$ & $<100$ & $>300$ & 3 & 3 \\
$\mathrm{H}$ & $\varnothing 18,0 \mathrm{~mm}$ & $1750 \mathrm{~L} / \mathrm{min}$ & $140 \mathrm{~mL} / \mathrm{t}$ & $<100$ & $>300$ & 3 & 3 \\
\hline
\end{tabular}

\section{RESULTADOS E DISCUSSÃO}

Conforme mostra a Tabela 4 e a Figura 3 optou-se por realizar o presente estudo para os aços tipo médio teor de carbono já que estes são os mais susceptíveis a ocorrência de romboidade. De acordo com a figura 3, no ano de 2012 na ArcelorMittal Cariacica, cerca de 234 toneladas de material foram sucateadas devido à romboidade, sendo que os aços de médio teor de carbono representam $59,4 \%$ desse total, com aproximadamente 139 toneladas. Em 2013, houve um aumento da perda de material devido à romboidade, com mais de 288 toneladas sucateadas; desse total, $73,4 \%$, ou seja, pouco mais de 211 toneladas, foram sucateadas. 
Tabela 4. Faixas de composição química para os aços de médio teor de carbono utilizados nos experimentos.

\begin{tabular}{ccccc}
\hline$\% \mathrm{C}$ & $\% \mathrm{Mn}$ & $\% \mathrm{Si}$ & $\% \mathrm{P}$ & $\% \mathrm{~S}$ \\
\hline $0,250-0,480$ & $0,600-0,700$ & $0,100-0,200$ & 0,030 máx & 0,030 máx \\
\hline
\end{tabular}
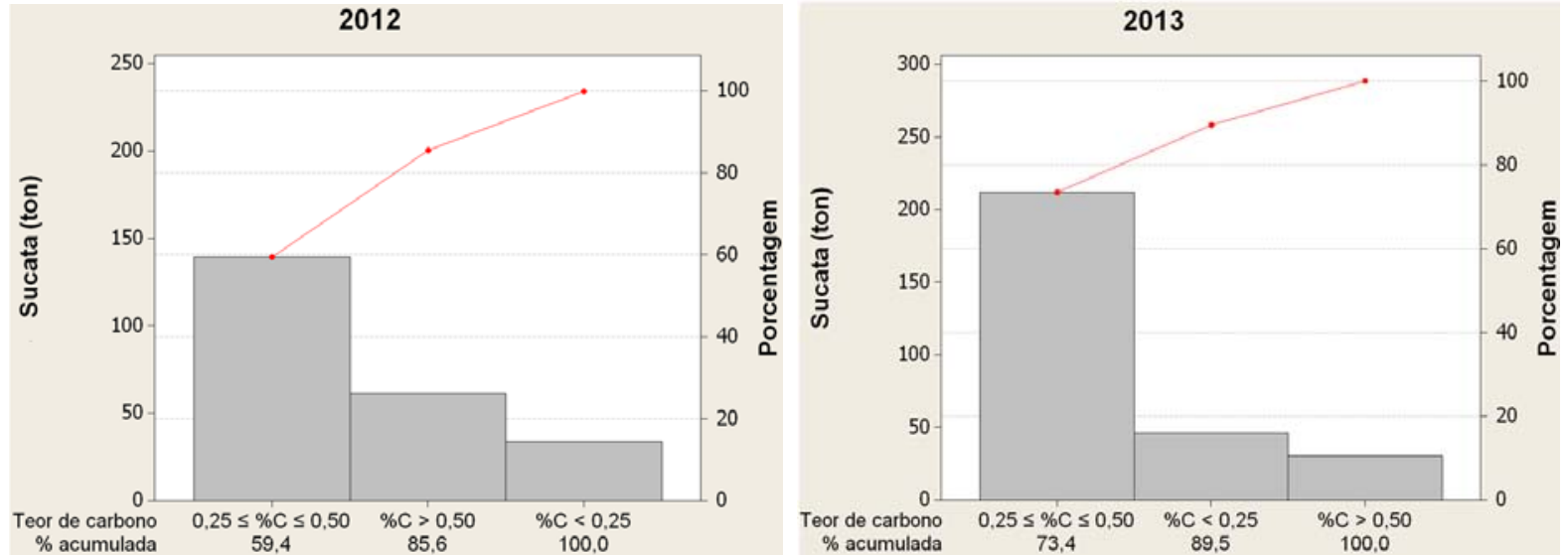

Figura 3. Gráficos de Pareto que relacionam a quantidade de sucata devido à romboidade pela categoria de aço nos anos de 2012 e 2013.

A figura 4 mostra um gráfico boxplot para as romboidades em cada veio. Analisando-se os valores das medições das diagonais foi possível verificar não existe tendência de formação do defeito em um veio em especial ou seja, não há uma variação significativa entre os valores de romboidade para os diferentes veios. Essa constatação é fundamental, pois mostra que nenhum dos veios é mais propenso à incidência de romboidade do que outro, logo, o veio de lingotamento não é um ruído no experimento. Vale ressaltar que todos os veios apresentaram, em algum momento, romboidades superiores a $16 \mathrm{~mm}$, sendo que no veio 1 a diferença das diagonais chegou a $19 \mathrm{~mm}$. A partir disso, para a realização dos testes, foram escolhidos os veios centrais (veios 2 e 3 ). A opção pelos veios centrais é justificada pelo fato de que os veios das extremidades (veios 1 e 4) são isolados preferencialmente caso ocorra algum atraso durante o processo, o que interromperia os testes, inviabilizando os experimentos.

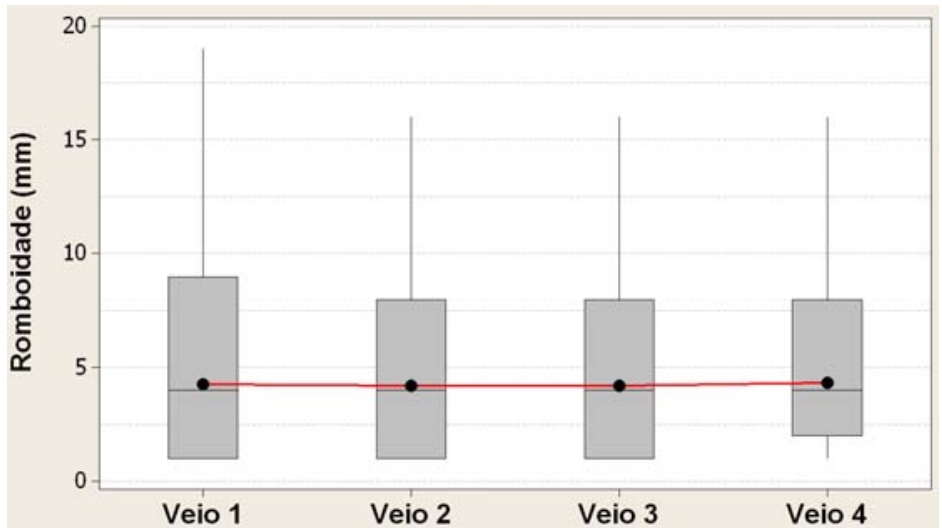

Figura 4. Boxplot para romboidade de aços de médio teor de carbono nos anos de 2012 e 2013.

A partir dos valores de romboidades dos experimentos 1, 2 e 3, foram calculados os valores médios e os respectivos desvios-padrão das romboidades para cada teste. Esses resultados, que são apresentados nas tabelas 5,6 , e 7, foram utilizados para realizar a análise estatística no MINITAB $^{\circledR}$. 
Tabela 5. Resultados para as medições de romboidade do experimento 1.

\begin{tabular}{lcccccccc}
\hline Teste & A & B & C & D & E & F & G & H \\
\hline Média das romboidades & 2,81 & 3,92 & 6,94 & 6,13 & 8,75 & 11,13 & 6,21 & 5,63 \\
Desvio-padrão das romboidades & 0,66 & 0,72 & 0,57 & 0,62 & 0,68 & 1,25 & 0,66 & 0,50 \\
\hline
\end{tabular}

Tabela 6. Resultados para as medições de romboidade do experimento 2.

\begin{tabular}{lcccccccc}
\hline Teste & A & B & C & D & E & F & G & H \\
\hline Média das romboidades & 3,25 & 6,13 & 9,19 & 5,45 & 5,69 & 8,44 & 4,95 & 3,00 \\
Desvio-padrão das romboidades & 0,68 & 0,62 & 0,66 & 0,69 & 0,70 & 0,63 & 0,76 & 0,65 \\
\hline
\end{tabular}

Tabela 7. Resultados para as medições de romboidade do experimento 3.

\begin{tabular}{llcccccccc}
\hline Teste & & A & B & C & D & E & F & G & H \\
\hline \multirow{2}{*}{ Veio 2 } & Média das romboidades & 3,67 & 4,00 & 7,33 & 5,63 & 4,50 & 5,44 & 2,33 & 0,83 \\
& Desvio-padrão das romboidades & 0,65 & 0,89 & 0,89 & 0,89 & 0,53 & 0,73 & 0,98 & 0,58 \\
\hline \multirow{2}{*}{ Veio 3 } & Média das romboidades & 7,00 & 7,63 & 10,69 & 9,06 & 6,50 & 7,10 & 5,58 & 3,75 \\
& Desvio-padrão das romboidades & 0,74 & 0,72 & 1,08 & 1,00 & 0,76 & 0,72 & 0,79 & 0,97 \\
\hline
\end{tabular}

Ao realizar a análise estatística no Minitab, o gráfico de Pareto para os efeitos mostrou que, para os experimentos 1 e 2, a vazão de água no molde e a válvula calibrada de troca rápida foram os fatores que mais afetaram a variável resposta romboidade, como mostra a figura 5. Para o nível adotado, não houve significância para a taxa de lubrificação (experimento 1) e para o superheat (experimento 2).

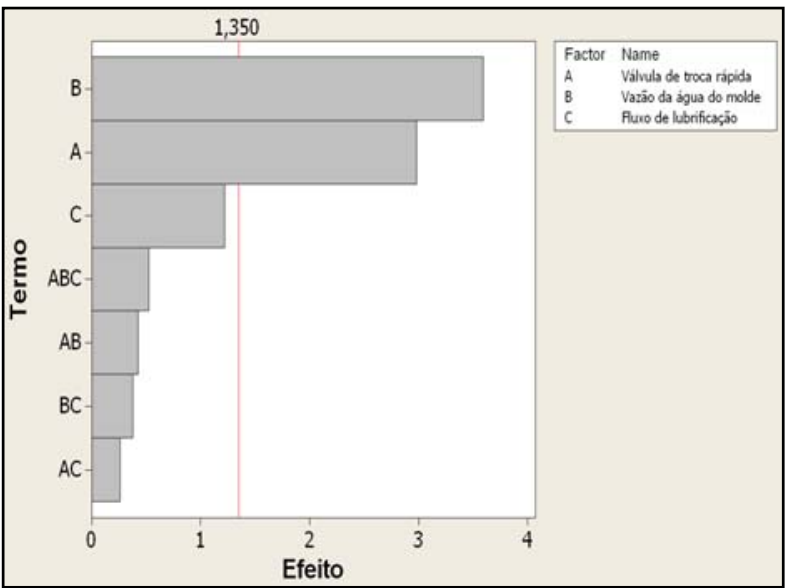

(a)

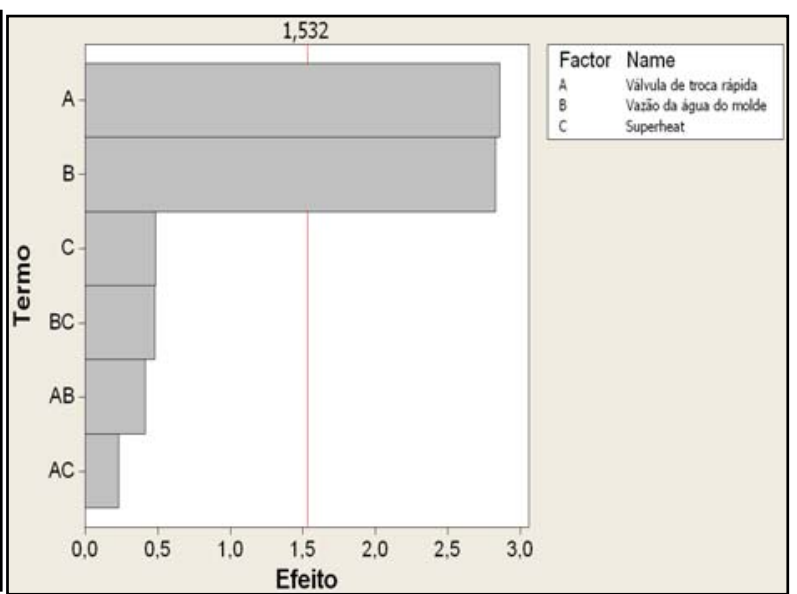

(b)

Figura 5. Gráfico de Pareto para a romboidade média: (a) experimento 1 e (b) experimento 2.

O gráfico multi-vari permite avaliar as interações entre os três fatores testados, bem como as implicações causadas pelas mudanças de níveis entre os fatores. A partir da figura 6 , verifica-se que os melhores resultados para os experimentos 1 e 2 , ou seja, as menores romboidades, são encontradas quando a vazão da água de refrigeração do molde é $1750 \mathrm{~L} / \mathrm{min}$, a válvula calibrada de troca rápida é de $\varnothing 18,0$ $\mathrm{mm}$ e o fluxo de lubrificação é de $140 \mathrm{~mL} / / \mathrm{t}$ (experimento 1) e o superheat é baixo (experimento 2). No experimento 2 verifica-se que a mesma tendência é observada porém a exceção ocorre para o superheat no qual o nível mais baixo apresenta menor romboidade, porém, nesta situação a influência do superheat é pouco relevante. A corrida na qual foi realizada o experimento 1, por atrasos no processo, foi lingotada em três veios e, por isso, liberada pelo forno panela com temperatura elevada (em torno de $1600^{\circ} \mathrm{C}$ ). Provavelmente, essa condição, associada ao menor nível de vazão de água de refrigeração do molde (1500 L/min), que proporciona 
velocidade de passagem de água no canal de refrigeração menor, possibilitou a fervura intermitente de água no canal de refrigeração. Entretanto, não é possível afirmar tal fato, pois não foram realizados ensaios de dureza para avaliar o amolecimento das paredes do molde.

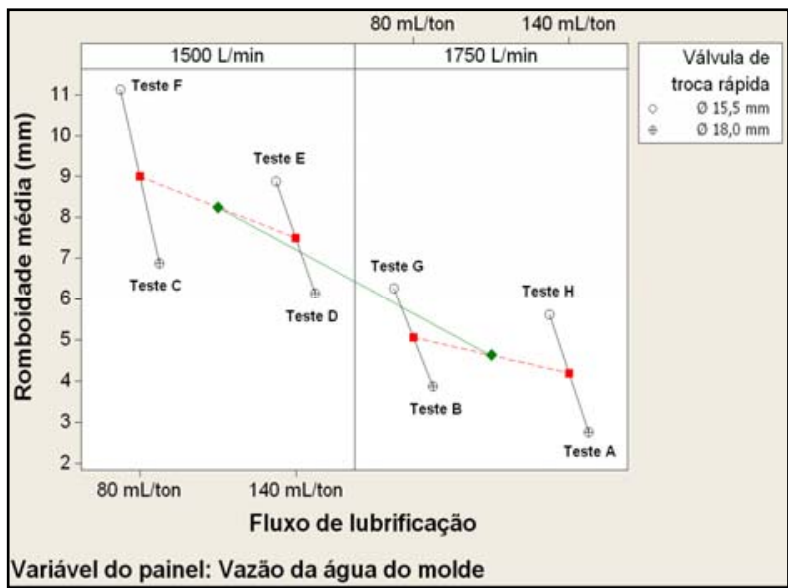

(a)

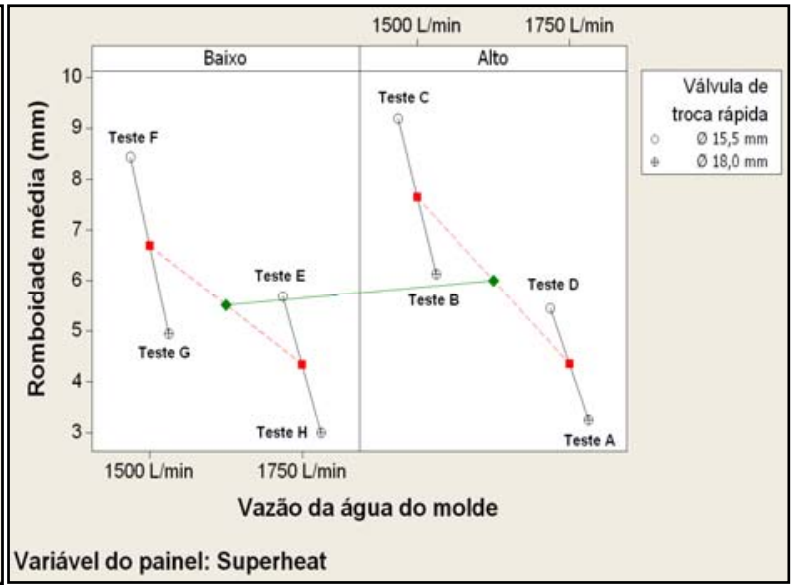

(b)

Figura 6. Gráfico multi-vari para a romboidade média: (a) experimento 1 e (b) experimento 2.

Diferente do proposto na literatura [3], o experimento 1 mostrou que a utilização de taxas de lubrificação mais elevadas, entre $42-58 \mathrm{~mL} / \mathrm{min}$ reduziu a romboidade. Verificou-se, com o auxílio do ibaAnalyzer (supervisório), que houve elevada variação de nível de metal no molde (entre 30 e $70 \%$ ). Tal fato, associado a operações de "molde quente", proporciona variações significativas na transferência de calor, as quais levam a diferenças na espessura da casca solidificada e contribuem para a incidência de romboidade. Essas variações na transferência de calor são provocadas tanto pela vaporização quanto pela escassez de óleo lubrificante, logo, maiores taxas de lubrificação contribuirão para a manutenção do filme de óleo líquido distribuído ao longo das faces internas do molde. No caso do experimento, contribuíram para operação de "molde quente" a elevada temperatura do aço e a baixa velocidade de passagem de água no canal de refrigeração.

Para o experimento 3, ao realizar a análise estatística no Minitab, o gráfico de Pareto para os efeitos mostrou que todos os fatores afetaram a variável resposta romboidade, conforme pode ser visto na figura 7 .

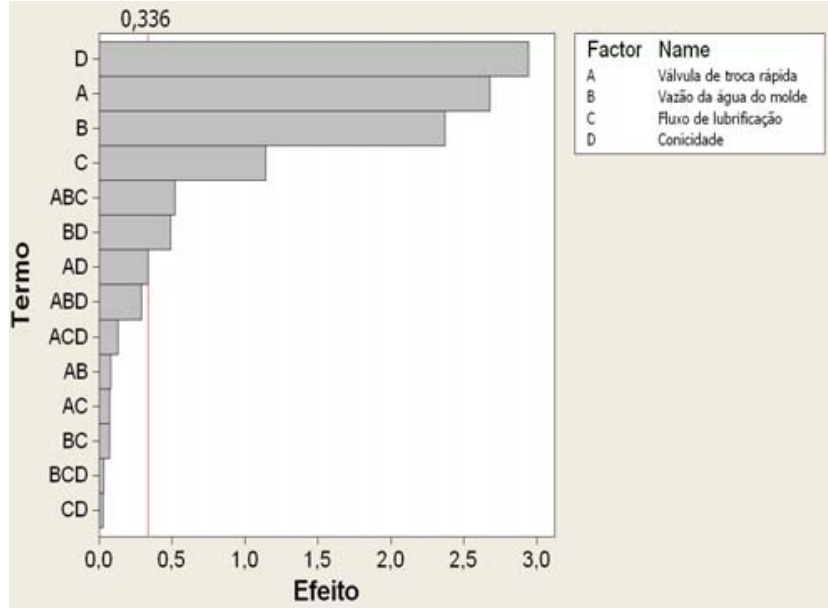

Figura 7. Gráfico de Pareto para a romboidade média referente ao experimento 3. 
O gráfico multi-vari apresentado na figura 8 mostra que as menores romboidades, foram encontradas para o teste $\mathrm{H}$ do veio 2. Por outro lado, os piores valores de romboidade ocorreram no teste $C$ do veio 3 . Esses resultados, com exceção da conicidade, são semelhantes aos obtidos no experimento 1.

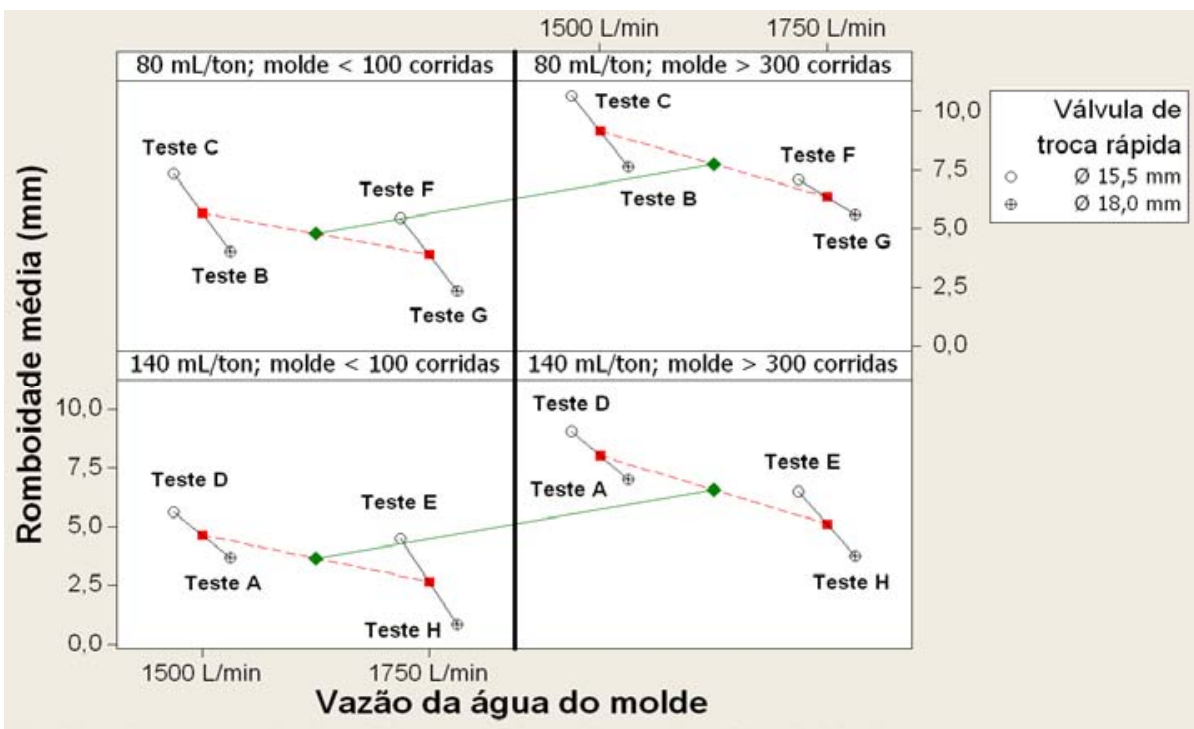

Variáveis dos painéis: Fluxo de lubrificação, Conicidade

Figura 8. Gráfico multi-vari para a romboidade media referente ao experimento 3.

Por fim, a conicidade mostrou-se o fator mais influente para a redução da romboidade. A utilização de moldes com maior número de corridas (no caso do experimento 3, mais de 300 corridas) contribuiu para o aumento das ocorrências e da gravidade da romboidade devido ao incremento do gap de ar entre metal e molde. Esse incremento é provocado pela suavização da conicidade nominal do molde e pelo desgaste tanto do recobrimento superficial quanto das paredes internas do molde, os quais aumentam a suscetibilidade às distorções térmicas que podem levar o molde à forma romboide. Na figura 9 é possível comparar as medições de conicidade para as faces paralelas e curvas feitas com TE-SiMM ${ }^{\circledR}$, que é um sistema de medição do perfil, conicidade, desgaste e deformação das faces e quinas do molde das máquinas de lingotamento contínuo.

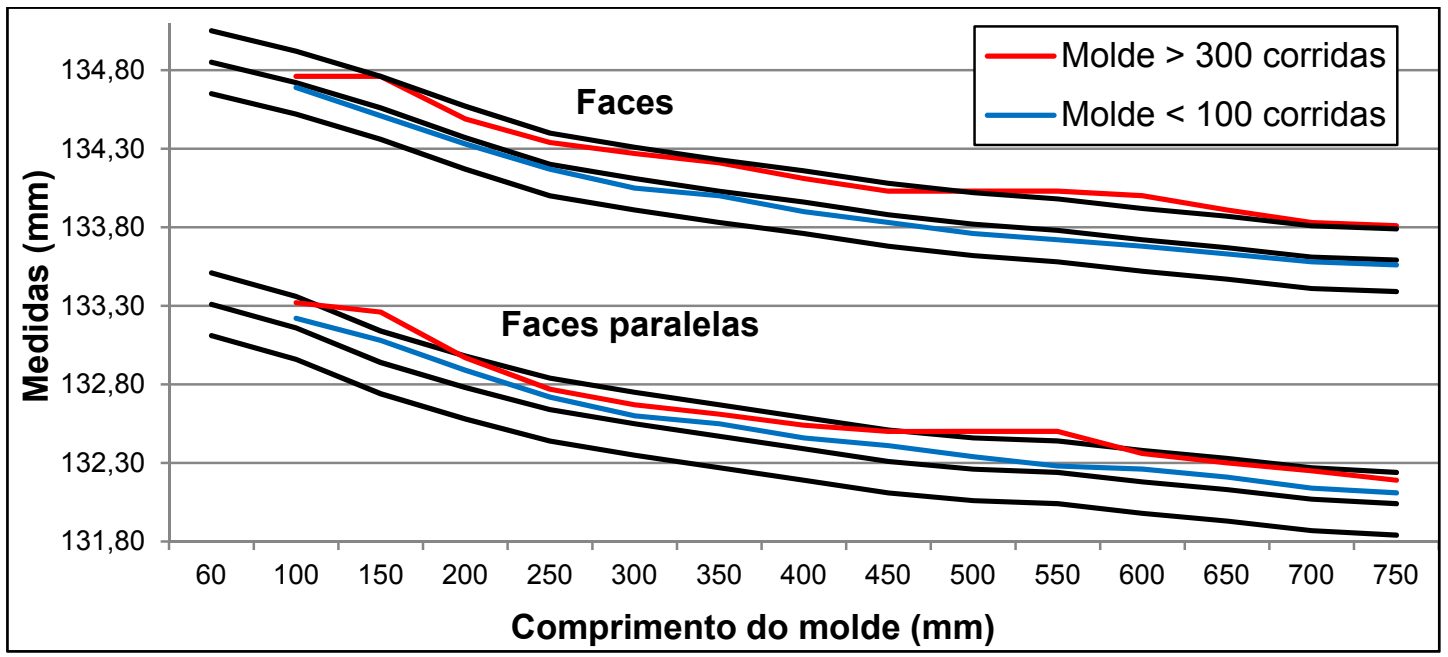

Figura 9. Medições de conicidade para os moldes utilizados no experimento 3. 


\section{CONCLUSÃO}

Baseando-se nos resultados obtidos e discutidos no presente trabalho, pode-se concluir que:

- A melhor condição para a minimização da romboidade ocorre quando a vazão da água de refrigeração do molde é $1750 \mathrm{~L} / \mathrm{min}$, a válvula calibrada de troca rápida é de $\varnothing 18,0 \mathrm{~mm}$, a o fluxo de lubrificação é $140 \mathrm{~mL} / \mathrm{t}$ de aço, o superheat é baixo e o molde apresenta menos de 100 corridas. Neste caso, a média e o desvio-padrão das romboidades foram, respectivamente, 0,83 e 0,58 $\mathrm{mm}$. Em contrapartida, a pior condição ocorre para a condição inversa, na qual a média e o desvio-padrão das romboidades foram, respectivamente, 10,69 e 1,08 mm;

- A conicidade mostrou-se o fator mais influente e a utilização de moldes com menor número de corridas contribuiu para reduzir a romboidade em até $3,5 \mathrm{~mm}$;

- Maiores velocidades de lingotamento contribuem para a melhoria do processo, reduzindo a romboidade em até $3 \mathrm{~mm}$;

- A utilização de taxas de lubrificação mais elevadas contribuiu para a redução da romboidade em até $1 \mathrm{~mm}$;

- Os resultados obtidos para o superheat estão em concordância aos da literatura. Entretanto, esse fator mostrou pouca influência, contribuindo em menos de $1 \mathrm{~mm}$ para a redução da romboidade;

- O planejamento e análise de experimentos mostrou-se muito útil para avaliação das relações entre fatores e suas influências na variável resposta romboidade, sendo um instrumento eficaz para otimização e melhoria de processos industriais.

\section{REFERÊNCIAS}

1 Kumar, S. Mould thermal response and formation of defects in the continuous casting of steel billets. 1996. $310 \mathrm{f}$. Tese. Departament of Metals and Materials Engineering, University of British Columbia, Vancouver, 1996.

2 Kumar, S. An expert system to diagnose quality problems in the continuous casting of steel billets. 1991. 199 f. Dissertação. Departament of Metals and Materials Engineering, University of British Columbia, Vancouver, 1991.

3 Brendzy, J. L.; Bakshi, I. A.; Samarasekera, I. V.; Brimacombe, J. K. Mould-strand interaction in continuous casting of steel billets Part 2. Lubrication and oscillation mark formation. Ironmaking and Steelmaking, v. 20, n. 1, p. 63-74, January-February 1993.

4 Chow, C. The effects of high speed casting on the mould heat transfer, billet solidification, and mould taper design of continuously cast steel billets. 2001. $182 \mathrm{f}$. Tese. Departament of Metals and Materials Engineering, University of British Columbia, Vancouver, 2001.

5 Chandra, S. Heat transfer, oil lubrication and moulds tapers in steel billets casting machines. 1992. 325 f. Tese. Departament of Metals and Materials Engineering, University of British Columbia, Vancouver, 1992. 